\title{
A Mobile Application for motor coordination deficiency users
}

\author{
Roseli Aparecida Francelin Romero \\ Tiago Miranda Leite \\ rafrance@icmc.usp.br \\ tiago.miranda.leite@usp.br \\ Instituto de Ciências Matemáticas e de Computação \\ Universidade de São Paulo (USP) \\ São Carlos, SP, Brasil
}

\begin{abstract}
It is not uncommon to find people with manual difficulties due to problems of motor coordination or some physical or neurological deficiency. This can hamper, among other things, the learning of writing digits and letters of the alphabet. In this article, we present a tool, developed in Python, to be used in tablets or phones with Android system. The tool can help users with some kind of hand handicap in the learning of letters or numbers writing, through their interaction with the electronic device. This system uses a CNN for recognizing the letters or digits and to give a feedback positive or negative for the user. Further, it can help users to learn about the corresponding image and sound, for each respective letter or digit. The mobile application is easy to use and has been tested in the learning of autistic children for 4 months. During training, the children demonstrated a good familiarity with the software and improved writing performance.
\end{abstract}

\section{KEYWORDS}

Deep Learning; Graphic User Interface; tool for training of the writing

\section{INTRODUCTION}

Mobile devices, such as, smartphones and tablets, has been increasingly present in people's daily life, being applied to a wide range of tasks, such as, shopping, trajectory planning, games, videos and more and more applications could be cited. Such devices has been used to train children in educational tasks, such as, the system developed by Gordon et al. [1] to learn a second language.

In [5], it can be found one of the first - and still one of the most important - studies about computers in Education. Papert presented the trends for computers in the educational context. Computers were a recent thing and their application in any subject still was in its first steps. It presents a reflection of how computers could influence the early education process. Autistic children can also learn vocabulary through a system proposed in [2]. There are also interactive games to identify objects and concepts through images and texts as in [7]. Game-Based learning strategy has been shown to be an enjoyable and effective strategy. Especially, when it comes to logic and math teaching, the use of robotic games contributes to achieving an effective educational synergy. Students from elementary school who learn in these conditions get better results

In: XVIII Workshop de Ferramentas e Aplicações (WFA 2019), Rio de Janeiro, Brasil Anais Estendidos do Simposio Brasileiro de Sistemas Multimidia e Web (WebMedia). Porto Alegre: Sociedade Brasileira de Computação, 2019.

ISSN 2596-1683

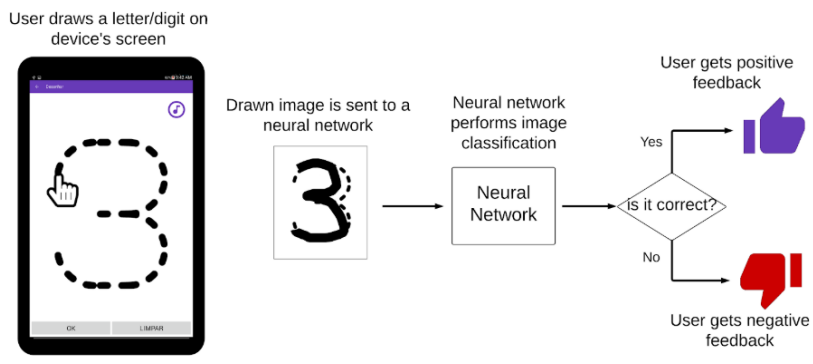

Figure 1: Components of the system

in programming classes, compared to students under a more traditional strategy. This type of challenge, besides motivating the students, is also an option to stimulate their imagination and, consequently, their cognitive skills [6]. Deep Learning networks, such as, convolutional neural networks (CNNs), are used to assist a child in the identification of facial expressions [8]. AlexNet was one the first models of CNN to classify the Imagenet database (containing approximately more 1 million of labeled images), outperforming classical computational vision algorithms [4].

In this work, a mobile application has been developed for training people with some kind of motor coordination deficiency to write letters and numbers by using a mobile device. This educational application runs in smartphones and tablets, allowing the identification and evaluation of images containing the objects mentioned.

\section{PROPOSED SYSTEM}

In this system, $\mathrm{CNN}$ is used for training the objects (letters and numbers) and an interface has been developed that provides: a correct signal, when the object drawn by the user is expected and wrong signal otherwise. Further, the system also provides the sound corresponding to the letter or number focused. So, through this system is possible to train writing and know the respective sounds of the object. The application receives an image, containing a digit or letter of the alphabet, through an interface, which establishes communication between the user and the application. An interface communicates with the pattern recognition module to check whether a letter or a digit was created correctly by the user. The response module emits a response, showing a finger (red hand). The application's response indicates whether the user has correctly written a number or letter, or not. In Figure 1, it is shown the components of the proposed system. The components that constitute 


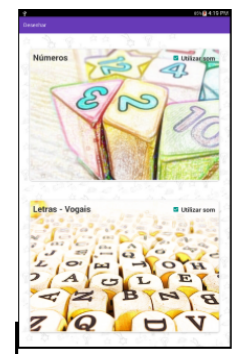

Figure 2: Initial Screen of the interface

the system will be presented in more detail to follow.

\subsection{Interface}

The interface of the digits and letters training application was developed using the Java language in the Android Studio application development environment in version 3.2. The user, by clicking the application icon installed on their tablet, can observed the application start screen, shown in Figure 2.

The user can choose between drawing numbers (digits) and vowels as desired. For each of these two options, a new screen is displayed, respectively, with a list of options of the 10 different digits in our numeric system (the decimal) and the 5 vowels of our alphabet. Clicking on the desired option starts a user's interaction session with the application. Thus, initially, a screen is presented showing the format, in dashed lines, of the character to be drawn by the user as it is shown in Figure 3. At the same time, the icon of a small hand moves through the screen, indicating the correct movement to be made by the user during the process of drawing the symbol. Then the user can slide his finger on the screen in an attempt to fill the dashed figure, drawing the letter or digit chosen.

The purpose of this procedure is to provide to the user the training of the format of the letters and/or digits during the filling of dashed lines, that are becoming more faded at each iteration, trying to induce the user, after being trained, to carry out the drawing of the letter and/or digit without assistance of the software. In Figure 3 is shown a screen with symbol of the number 3 being filled by the user. After completing the dash, clicking the "OK" button sends the drawn image to the neural network present internally in the application, which performs the figure classification process, instantly providing feedback to the user, indicating whether the object concerned was designed correctly or not. There is also the option to clean the image if one wants to erase the drawing and then redo it.

Although in Figure 3, there are two options to be chosen between numbers and vowels, it is possible to extend the interface to providing also the option to consonant letters. The consonant letters could be trained following the same procedure as it was applied to train the vowels and numbers as it is described in next section.

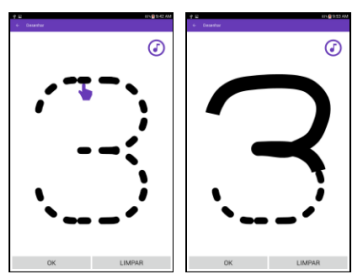

Figure 3: Screen of users interaction with the application

\subsection{Pattern Recognition Module}

A deep neural network, known as CNN (Convolutional Neural Network), is used in this work to classify the image provided by the user. It consists of 4 layers of convolution 2D, with function of activation ReLU, and a layer totally connected, also with function of activation ReLU. In the last layer of the network, it was used softmax activation function, responsible for making the final classification of the image. It provides as output a distribution of probabilities among the 15 classes that the network recognizes, such as, the aforementioned 10 digits and the 5 vowels. Dropout is used in the weights of the fully connected layer during training, since this has been shown to be an efficient technique to avoid over-adjustment of the model. For training of $\mathrm{CNN}$, two databases were used. It was used MNIST dataset ${ }^{1}$ for training the numbers and the $74 \mathrm{~K}$ dataset $^{2}$ for training letters of alphabet. As it was mentioned before, the consonant letters could be trained following the same procedure explained here. The proposed $\mathrm{CNN}$ architecture can be seen in Figure 4.

The network training used, in each iteration, lots of 100 samples of randomly selected images. One step corresponds to a complete training cycle in which all batches have been used. Thus, the total duration of training of the network corresponded to 20 epochs. The cost function used was cross-entropy, which is currently used in classification problems. For optimizing of the rate learning, it was used Adam Algorithm [3]. At the end of the training, the entire structure of the neural network, as well as its weights, were saved as a file and then sent to the Android application.

Although they are different development environments, it is possible to use in Android (Java) a neural network model that has been implemented in Python. In order to do this, it is necessary to import the Java language TensorFlowInferenceInterface class which, besides being able to read the network that was saved to a file, can also be used to make inferences, being the main part of the Pattern Recognition Module.

Thus, after receiving the image of the drawn symbol as input, this class returns, through a call of the method fetch( ), an array of 15 positions, representing the classification layer of the network, filled with values between 0 and 1 , which indicate the probability of the image in question belongs to each one of the 15 classes.

\subsection{The Result Emission Module}

This module consists of a Java class implemented in order to verify the relationship between the expected result and that obtained

\footnotetext{
${ }^{1}$ Available at: http://yann.lecun.com/exdb/mnist/

${ }^{2}$ Available at: http://www.ee.surrey.ac.uk/CVSSP/demos/chars74k/
} 


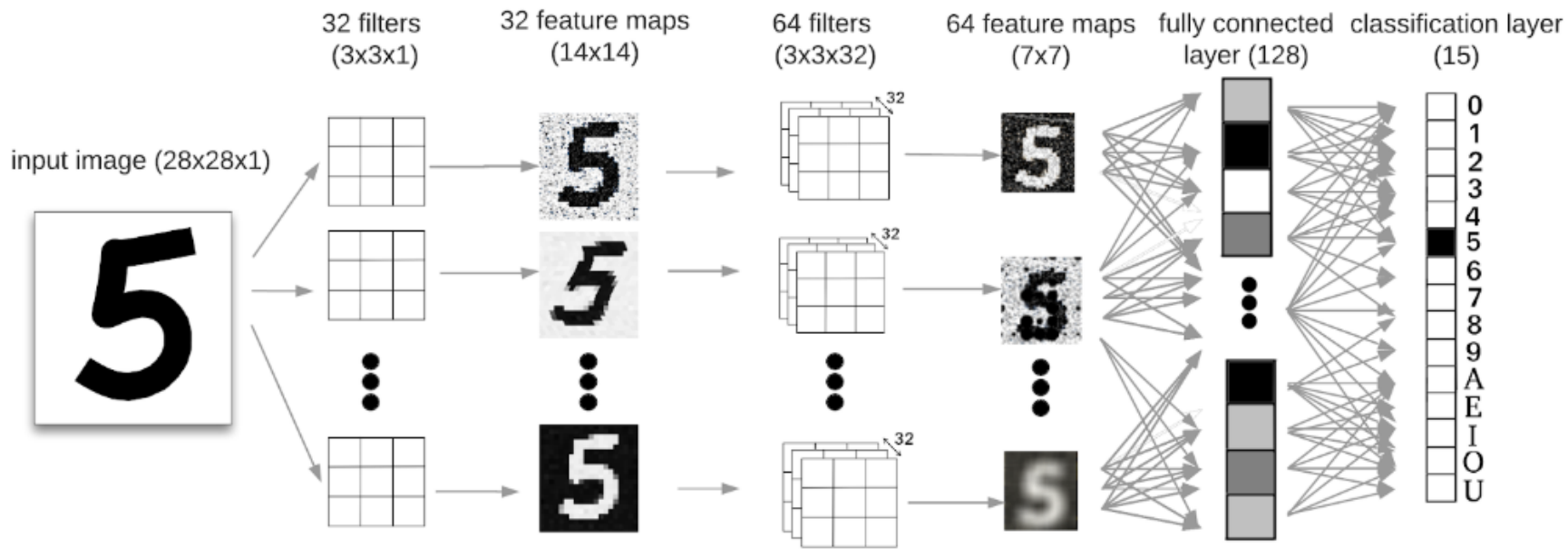

Figure 4: Architecture of CNN

by the Pattern Recognition Module, aiming to provide feedback to the user. Such a class has a variable indicating the expected character, and thus compares it with the highest probability symbol returned by the Pattern Recognition Module. If both are different, the application has an error, displaying a red hand icon, flagging it negatively, and allowing the user to try again. Otherwise, the system reports a hit, showing a blue hand icon positively signaling.

\subsection{Experiments}

Several experiments were conducted with 3 children of APAE Institution, diagnosed with some degree of autism. with the proper authorization of the parents. Data were collected during a period of 4 months, with weekly visits, to be performed an analysis of the results. In relation to the developed interface, the students did not present problems in using it; they were naturally familiar with the application, showing good interaction with it, which shows a positive point.

Students' performance was measured according to the degree of difficulty showed. When drawing each digit on the application screen, three cases were considered. It was recorded whether the student was able to do it without the teacher's help or he needed help or if it was not able to finish the drawing. The total number of occurrences of each of these three difficulty levels was then counted, and their frequency was then calculated. The three students were asked to draw the digits from 1 to 9 , repeating each symbol several times.

Finally, to test if there was improvement in children's motor coordination, experiments were performed on sheets of paper. Thus, at the end of the training period with the application, the children were asked to make a sketch of the same numbers previously trained, this time on A4 size sheet.

\subsection{Discussion of Results}

In this section the performance of the children in using the proposed application, taking into account the frequency of each outcome, is discussed. The performance of the student A, considering the

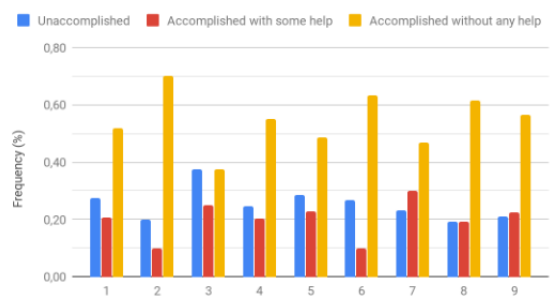

I

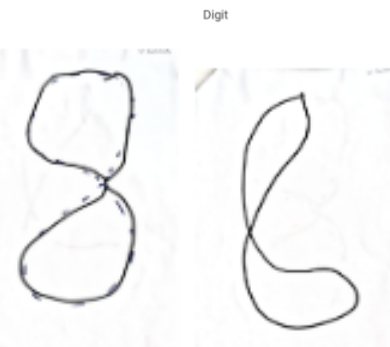

Figure 5: Student As performance, on the left and drawing of number written at first and second attempts, on the right.

frequency of occurrence of each degree of difficulty manifested by them, are presented in Figure 5.

According to the graph of Figure 5, it is noted that since the beginning of the training the student showed difficulty in circumventing numbers 7 and 8, for example. After the training, there was an improvement in the contours of these figures, since the student was able to draw them correctly in the first and second attempt, without the dash, as it can been in this figure.

In the case of student B (Figure 6), it is noted that since the beginning of the training he already had difficulty in circumventing numbers 2 and 9. After the training, a slight improvement in the contours of these was observed, being the student able to draw the 


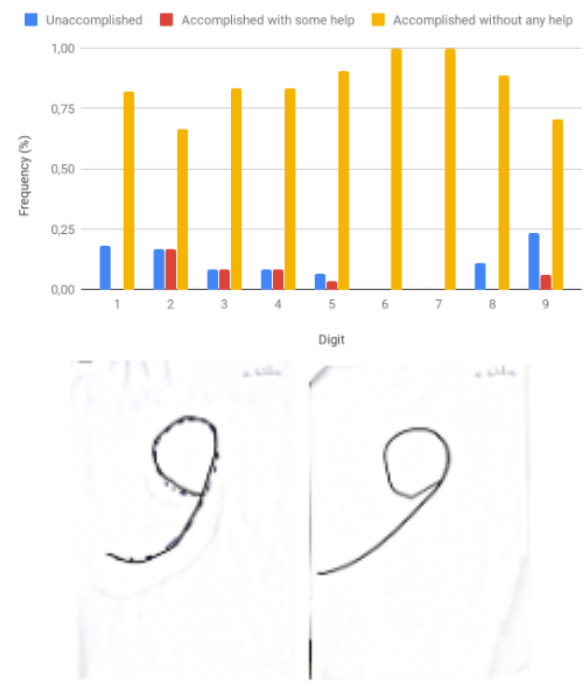

Figure 6: Student Bs performance on the left and drawing of number written at first and sixth attempts, on the right.

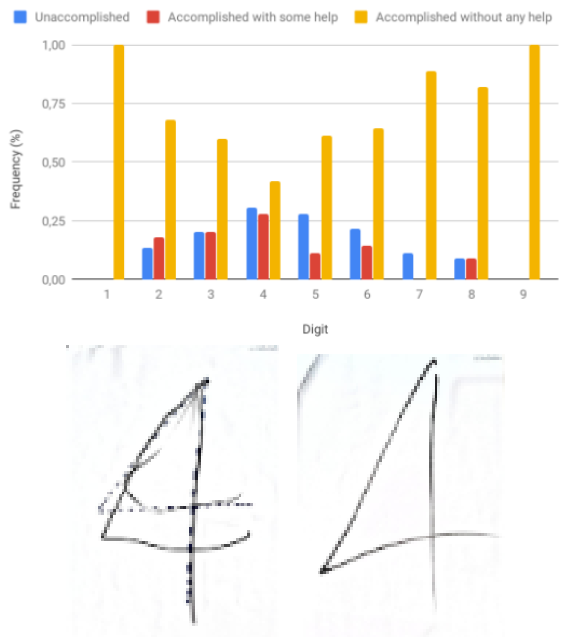

Figure 7: Student Cs performance on the left and drawing of number written at first and fifth attempts on the right.

number 9 correctly and without the dashed, in the white paper, at the first and sixth attempt, shown in this sequence in this figure.

In the case of student $C$, whose performance is shown in Figure 7 , it was noted a difficulty to circumvent the number 4 . After the training, the student was able to do this number manually, without the dash, in the fifth attempt.

The analysis of the results showed a small improvement in these motor coordination of the children, regarding the writing of the numbers, after 4 months of training in weekly sessions. This provides an indication that with a higher frequency and training time, better results could be obtained. A demonstration of the system interface can be accessed in https://drive.google.com/open?id=1U-CJL9FgZhEH0MbSBbYFdgYD-bWIj3ah.

\section{CONCLUSION AND FUTURE WORK}

In this article, a training tool for letters and digits writing was proposed. A Deep Learning network, CNN, considered one of the most promising techniques for pattern recognition was used for object recognition. A good generalization capability was achieved with only 4 convolutional layers, turning it feasible to use in systems with limited processing and memory capacity, such as mobile devices and tablets. It has also been noted that the latency time of the network is imperceptible, providing instantaneous response. The application was successfully used in experiments carried out with students of APAE Institution, diagnosed with autism and writing difficulties due to lack of motor coordination, in order to directly evaluate the impact of the technology developed in the learning of writing of autistic children. The experiments were carried out during 4 months. The tool developed was very well accepted by the children and it was noted a small improvement in writing on the sheet after training.

In spite of the experiments were done with autistic children, the application can be useful to other users, such as in physiotherapy sessions with patients recovering of fine movements of their hands and for elderly people losing the moving of their hands. This system not only can provide the training of the writing, but also for the image recognition and sound of letters e/or digits and in this way, it could be also used in educational tasks.

In the interface tested, only vowels (among letters possible) and digits were considered. It is is part of the future work to extent the system to offer the possibility for training the writing of all letters of alphabet. Furthermore, a long interaction analysis is necessary considering a greater number of users for a more definitive conclusion.

\section{REFERENCES}

[1] Gordon D. A. Brown. 1992. Cognitive Psychology and Second Language Processing: The Role of Short-Term Memory. Advances in Psychology 83 (1992). https://doi.org/10.1016/S0166-4115(08)61490-9

[2] Budianingsih Emir Husni. 2013. Mobile applications BIUTIS: Let's study vocabulary learning as a media for children with autism. Procedia Technology. 11 (2013), $1147-1155$.

[3] Diederik P. Kingma and Jimmy Ba. 2014. Adam: A Method for Stochastic Optimization. arXiv e-prints. (2014), p. arXiv:1412.6980.

[4] I.; HINTON G. E. KRIZHEVSKY, A.; SUTSKEVER. 2012. Imagenet classification with deep convolutional neural networks. Advances in neural information processing systems. [S.l.: s.n.] (2012), 1097-1105.

[5] S. M. Papert. 1985. Logo: Computadores e Educação. São Paulo, Editora, Brasiliense. (1985).

[6] Jaekwoun Shim, Daiyoung Kwon, and Wongyu Lee. 2017. The Effects of a Robot Game Environment on Computer Programming Education for Elementary School Students. IEEE Transactions on Education 60, 2 (2017), 164-172.

[7] Sourav Suresh ; Varun Nagaraj Rao ; Gowri Srinivasa. 2018. Gamification of a visual question answer system. Proc. 2018 IEEE Tenth International Conference on Technology for Education (T4E). (2018), 41-44. https://doi.org/10.1109/T4E. 2018.00016

[8] Md Inzamam Ul Haque; Damian Valles. 2018. A facial expression recognition approach using DCNN for autistic children to identify emotions. 2018 IEEE 9th Annual Information Technology, Electronics and Mobile Communication Conference (IEMCON). (2018), 546-551. https://doi.org/10.1109/IEMCON.2018. 8614802 